\title{
A Silent and Debilitating Case of Obstructing Hydrocephalus
}

\author{
Alex Zaharcu*, Rohit Kumar, Mark Phillip Baker, John Guirgis, Furqan Haq and Nemer Dabage \\ Department of Internal Medicine, Blake Medical Center, Bradenton, Florida, USA
}

\begin{abstract}
Background: Leptomeningeal metastases are complications of advanced cancer and have a scarce but often death-dealing incidence. Diagnosis often requires a good measure of suspicion and patients can present with a vast diversity of signs and symptoms.

Case presentation: A 76-year-old male who comes in with altered mental status of unknown etiology with past medical history significant for multiple types of cancer and recent travel to Vancouver. He was investigated by our internal medicine team for altered mental status, pulmonology for a possible pneumonia, neurology for possible HSV encephalitis vs cranial metastases and infectious disease for a new onset rash on his torso.
\end{abstract}

Conclusion: Metastasis was diagnosed through spinal fluid cell analysis and patient was sent to hospice.

Keywords: Meningeal metastases; Lung cancer; Hydrocephalus

\section{Introduction}

Leptomeningeal carcinomatosis is found in $5 \%$ to $8 \%$ of patients with solid cancer and specifically $9 \%$ to $25 \%$ in lung cancers as our patient. We will exclude other data that is not pertinent to our case scenario.

There are two types of Leptomeningeal carcinomatosis: free floating cells that do not adhere and nodular contrast-enhancing tumors that bind to the organ wall and are pertinent on MRI with contrast.

Frequent symptoms of Leptomeningeal carcinomatosis are cranial nerve paralyses, non-specific headaches, back pain, hearing loss and neurocognitive syndromes [1].

The main tests that all patients with suspected Leptomeningeal Carcinomatosis should get are a spinal tap with cytology analysis and cranial/spinal MRI studies with contrast. Although a spinal tap with fluid analysis is definitive, it is only positive for cells in $50 \%$ to $60 \%$ of cases and repeat tap ads $85 \%$ to $90 \%$ sensitivity. At least $5-10 \mathrm{ml}$ of cerebral-spinal fluid should be taken and analyzed within one hour. MRIs can see lesions of adherent cells in $70 \%-80 \%$ [2]. A high protein and/or lactate concentration in the CSF may be regarded as indication for a high tumor burden in the CNS [3].

Detection of Leptomeningeal metastases in adenocarcinomas has some background evidence with a high sensitivity of CEA 91\% [4]. The diagnostic value of the vascular endothelial growth factor (VEGF) in the CSF is promising, but data are preliminary and not yet sufficient for routine application. If left untreated, the LM of solid tumors leads to death within about 4-6 weeks [5].

\section{Case Presentation}

A 76-year-old Caucasian male comes to our community hospital with continuous mental decline for the past two weeks. Past Medical History is pertinent for migraine headaches which started three months prior, previous Lung Cancer (Adenocarcinoma), Prostate Cancer and Head and neck cancer (left pharynx Squamous Cell Cancer) all surgically resected with no chemotherapy or radiation.

The patient had no prior debilitating symptoms and his mental status was intact before being admitted to our facility. Interestingly, before the migraines began he had made a trip out to Vancouver, was on Victoria Island, then went up to the Southeast Alaskan coast on a cruise (Fairbanks) and then flew home. For the past two weeks he started being confused and had troubles managing daily activates, everything culminating with loss of bowel and bladder control and poor balance with a subsequent fall onto his spouse.

Physical examination on presentation was pertinent for mild confusion on orientation to person and place but not time, CNII-XII grossly intact, extra ocular movements intact, pupils equal round and reactive to light, no meningismus sign, regular heart rate and rhythm and clear to auscultation bilaterally.

Table 1 lists the investigative studies with information of the chest radiograph, fungal culture, Histoplasma antigen and Venereal Disease Research Laboratory test. First Cerebral-Spinal fluid tap with a prominence of RBC due to traumatic injury upon patient movement (Table 2).

Table 2 shows the first collection of the Cerebral-Spinal fluid tap and its characteristic appearance, hematology and biochemistry. The table shows prominence of RBC due to traumatic injury upon patient movement.

Table 3 shows the second collection of Cerebral-Spinal Fluid and its characteristic appearance, hematology and biochemistry. There was no prominence of RBC as seen in the first collection.

\section{Patient's progress and outcome}

Patient arrived at our facility being mildly confused with orientation to person and place but not time. He was able to identify wife, be mildly alert and awake for the first part of admission.

He was admitted to the floor where his altered mental status decompensated up to a point where he required tube feedings. Neurology was consulted, and recommended spinal tap for CSF

*Corresponding author: Alex Zaharcu, Department of Internal Medicine, Blake Medical Center, PGY-2, 2020 59th street west, Bradenton, Florida, 34209, USA Tel: 941-545-6218; E-mail: Alexandru.zaharcu@hcahealthcare.com

Received: October 10, 2017; Accepted: December 22, 2017; Published December 27, 2017

Citation: Zaharcu A, Kumar R, Baker MP, Guirgis J, Haq F, et al. (2017) A Silent and Debilitating Case of Obstructing Hydrocephalus. Oncol Cancer Case Rep 3: 140.

Copyright: (c) 2017 Zaharcu A, et al. This is an open-access article distributed under the terms of the Creative Commons Attribution License, which permits unrestricted use, distribution, and reproduction in any medium, provided the original author and source are credited. 


\begin{tabular}{|c|c|c|c|c|}
\hline CXR & \multicolumn{2}{|c|}{$\begin{array}{c}\text { Fungal culture of CSF } \\
\text { and Herpes I and II }\end{array}$} & $\begin{array}{c}\text { Hystoplasma } \\
\text { Ag }\end{array}$ & VDRL \\
\hline CT Brain & Haemophilus influenzae & Legionella Ag & Bartonella Hensalae \\
\hline MRI Brain & Neisseria meningitidis & TB quantiferon & Bartonella Quintana \\
\hline $\begin{array}{c}\text { MRI } \\
\text { C-spine }\end{array}$ & Hystoplasma yeast & Anti - Hu Ri Yo & Cryptococcus ag \\
\hline TSH & Folate & Vitamin B12 & \multicolumn{2}{|c|}{ Methyl Malonic Acid } \\
\hline
\end{tabular}

Table 1: Investigative studies showing the chest radiograph, fungal culture, histoplasma antigen and venereal disease research laboratory test.

\begin{tabular}{|c|c|}
\hline \multicolumn{2}{|c|}{ Lumbar puncture 1 } \\
\hline Appearance & Clear \\
\hline WBC & 16 \\
\hline RBC & 296 \\
\hline Glucose & 23 \\
\hline Protein & 119 \\
\hline
\end{tabular}

Table 2: First collection of the cerebral-spinal fluid tap and its characteristic appearance, hematology and biochemistry.

\begin{tabular}{|c|c|}
\hline \multicolumn{2}{|c|}{ Lumbar puncture 2 } \\
\hline Appearance & Clear \\
\hline WBC & 0 \\
\hline RBC & 23 \\
\hline Glucose & 38 \\
\hline Protein & 73 \\
\hline
\end{tabular}

Table 3: Second collection of cerebral-spinal fluid and its characteristic appearance, hematology and biochemistry.

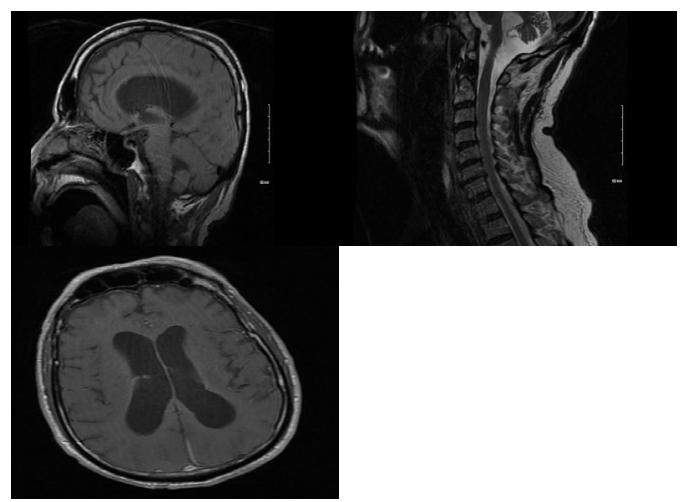

Figure 1: Normal pressure hydrocephalus on MRI with contrast and no trace of malignant adherent cells or cerebral masses.

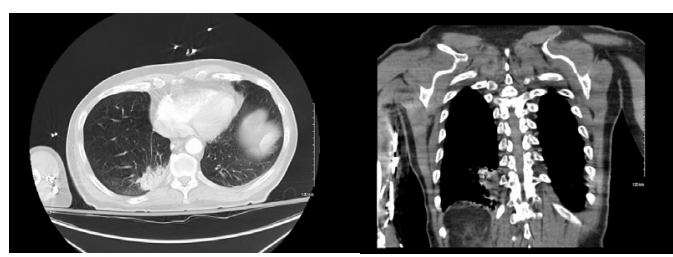

Figure 2: Posterior medial right lower lobe recurrent adenocarcinoma found on annual screening CT.

analysis for a suspected meningitis. CSF fluid came back positive for suspicion of viral, fungal or Tuberculous meningoencephalitis with normal opening pressure of $120 \mathrm{mmH}_{2} \mathrm{O}$. Infectious disease was consulted at this point.

Having had a subsequent rash on his chest and back, along with a previous travel history to Vancouver, Infectious Disease recommended screening for Cryptococcus and Histoplasmosis antigens. Patient was put on Acyclovir and Ceftriaxone /Vancomycin coverage for prophylactic treatment of disease until a clear cause was identified. Acyclovir was discontinued two days later due to rising creatinine and a concern for renal involvement.

While doing a CXR for tube feeding placement a right lower lobe PNA was suspected but could not be documented as aspiration or a previous infectious event. Neurosurgery suggested that a ventriculoperitoneal shunt might pose a benefit for the patient at this point given his ongoing hydrocephalus.

Having had prior lung cancer history, he was due for an annual screening Chest CT. Up until this point CT/MRI brain and spinal cord MRI did not elicit any acute ongoing process only a disproportionate enlargement of ventricles pointing to a communicating hydrocephalus.

CT chest with IV contrast showed a mass-like opacity in the posteromedial right lower lobe $4.5 \mathrm{~cm} \times 2.9 \mathrm{~cm} \times 3.7 \mathrm{~cm}$.

Concurrently with CT of chest, patient had a repeat spinal tap and before a biopsy of the lung mass would be undertaken the CSF fluid analysis showed multiple atypical cells which led to the diagnosis of Leptomeningeal Carcinomatosis. Family denied further interventions and patient was referred to hospice for comfort measures only.

Figure 1 shows the normal pressure hydrocephalus on MRI with contrast and no trace of malignant adherent cells or cerebral masses. CT chest with IV contrast showed a mass-like opacity in the posteromedial right lower lobe $4.5 \mathrm{~cm} \times 2.9 \mathrm{~cm} \times 3.7 \mathrm{~cm}$.

Figure 2 shows the posterior medial right lower lobe recurrent adenocarcinoma found on annual screening CT.

\section{Discussion}

We believe that this clinical vignette offers to the medical community a multifaceted and rare disease that needed management from a diverse team of physicians (Neurology, Infectious disease, Pulmonary, Internal Medicine, Hospice management). It masked itself as a neurological case which took the curious path of an atypical infectious process to finally being led in the right direction due to annual screening guidelines for Lung Cancer.

Our patient although he didn't have clear signs of meningitis (afebrile and no nuchal rigidity) we believe the second CSF analysis route was correctly followed due to previous history of cancer. The first spinal tap could not analyze cytology, but second tap was pertinent for atypical cells.

Previous travel history to Vancouver and sudden inpatient onset of peculiar skin rash spearing palms and soles made us consider an atypical infectious process. When our patient, only had increased WBC and no response to antiviral and antibiotic medication, fungal, TB or paraneoplastic processes were considered more in-depth.

Serial CXR while inpatient did not show any pulmonary masses and there was no need to do a chest CT up until now. Annual screening lung cancer screening and compliance was very helpful in our scenario. It led us to pinpoint our source of the metastatic disease and clarify it further with the CSF pathology analysis indicating multiple atypical cells.

Our patient was referred to hospice and evidence from the U.S. National Comprehensive Cancer Network (NCCN) supports the outcomes in our case. Poor prognosis is defined by NCCN consisting of low Karnofsky index ( $<60 \%$ to $70 \%)$, severe neurological deficits, parenchymal brain metastases and blockage of the CSF flow, extensive 
tumor involvement with little opportunity to response to systemic therapy $[6,7]$.

We are also trying to evaluate possible treatment modalities that would have extended our patient's period of survival or diminish his rapid mental decline.

Brain images showed hydrocephalus which could have been managed through a ventriculo-peritoneal shunt, a procedure which was considered by our neurology team but felt to be of minimal benefit, unlikely to result in the patient's awakening and offer no meaningful treatment response [1]

In order to obtain significant results with whole brain radiation therapy one would have to include in the procedure the cerebral meninges, cribriform plate, base of skull and spinal canal of the cervical vertebrae 1 and 2 . Survival periods after radiation therapy of the brain is still with a medium of 2 months. We could not establish clear foci of intracranial metastasis or meningeal occlusion sites throughout the whole CNS [1].

\section{Conclusion}

Whole brain radiation therapy might be beneficial in our case because it may restore circulation in up to $50 \%$ of cases, this further making room for intrathecal chemotherapy, but family did not want to further medical treatment.

Intrathecal drugs approved are Methotrexate, Cytarabine and Thiotepa. Advantages would be that these drugs obtain a better concentration in the CSF with bypass of the blood brain barrier. They also confer a lack of systemic involvement because of administration only in the CSF. Intrathecal chemotherapy can add a sustained benefit, albeit for a certain amount of time, but it can be a measure some might consider.

\section{Source(s) of support}

Graduate Medical Education at Blake Medical Center, Bradenton FL \& HCAWest Florida Division, Tampa, FL.

\section{References}

1. Mack F, Baumert BG, Schäfer N, Hattingen E, Scheffler B, et al. (2016) Therapy of leptomeningeal metastasis in solid tumors. Cancer Treat Rev 43: 83-91.

2. Prommel P, Pilgram-Pastor S, Sitter H, Buhk JH, Strik H (2013) Neoplastic meningitis: How MRI and CSF cytology are influenced by CSF cell count and tumor type. Sci World J.

3. Herrlinger U, Forschler H, Kuker W, Meyermann R, Bamberg M, et al. (2004) Leptomeningeal metastasis: Survival and prognostic factors in 155 patients. J Neurol Sci 223: 167-178

4. Moldrich G, Lange P, Strik H (2010) Carcinoembryonic antigen in the CSF of cancer patients-the value of intrathecal synthesis and correlation with IgAdiffusion dynamics. Acta Neurol Belg 110: 314-320.

5. Shapiro WR, Johanson CE, Boogerd W (2009) Treatment modalities for leptomeningeal metastases. Semin Oncol 36: S46-54.

6. Glantz MJ, Hall WA, Cole BF, Chozick BS, Shannon CM, et al. (1995) Diagnosis management, and survival of patients with leptomeningeal cancer based on cerebrospinal fluid-flow status. Cancer 75: 2919-2931.

7. Chamberlain MC, Kormanik PA (1996). Prognostic significance of 111indium-DTPA CSF flow studies in leptomeningeal metastases. Neurology 46: 1674-1677. 